\title{
Autoconcepto académico y motivación académica en jóvenes talento del departamento de Puno, Perú
}

\section{Academic self-concept and academic motivation in young talent from the department of Puno}

\author{
Mónica Cahuana Cuti \\ Universidad Peruana Unión. Juliaca, Perú \\ ORCID: https://orcid.org/0000-0001-9069-1068 \\ Oscar Mamani-Benito \\ Universidad Peruana Unión. Juliaca, Perú \\ ORCID: https://orcid.org/0000-0002-9818-2601 \\ Renzo Felipe Carranza Esteban \\ Universidad San Ignacio de Loyola, Lima, Perú \\ ORCID: https://orcid.org/0000-0002-4086-4845
}

Received 03-24-20 Revised 06-30-20 Accepted 12-10-20 On line 12-04-20

*Correspondence

Email: rcarranza@usil.edu.pe
Cite as:

Cahuana, M. Mamani-Benito, O., \& Carranza, E. (2020). Autoconcepto académico y motivación académica en jóvenes talento del departamento de Puno, Perú. Propósitos y Representaciones, 8(3), e788. doi: http://dx.doi.org/10.20511/pyr2020.v8n3.788 


\section{Resumen}

El objetivo del estudio fue determinar la relación entre las variables autoconcepto académico y motivación académica en jóvenes talento de Puno-Perú. La metodología corresponde al estudio de tipo correlacional, de diseño no experimental y corte transversal; se trabajó con una población de 80 universitarios beneficiarios del programa beca 18 de una universidad privada de Puno, siendo el $48.8 \%$ de sexo masculino y el $51.3 \%$ de sexo femenino, a quienes fue aplicado dos instrumentos de medición documental: la sub escala de autoconcepto académico AF5 y una escala de motivación académica, ambos válidos y confiables. Los datos fueron procesados con el paquete estadístico SPSS (Statistical Package for the Social Sciencies) versión 22.0 a un nivel de significancia del 5\% y nivel de confianza del 95\%. Los resultados según el coeficiente de correlación Rho de Spearman evidenciaron la existencia de una correlación moderada, directa y significativa entre las variables de investigación (Rho $=.497 ; \mathrm{p}=.000$ ) así como en las dimensiones motivación intrínseca (Rho $=.475, \mathrm{p}=.000)$ y motivación extrínseca (Rho $=.485$, $\mathrm{p}=.000$ ), en cambio en Amotivación se halló una correlación inversa baja y no significativa (Rho $=-.196, \mathrm{p}=.082)$. Se concluye que, a mayor autoconcepto académico, mayor será la motivación académica.

Palabras clave: autoconcepto académico, motivación académica, jóvenes talento, programa nacional Beca 18.

\section{Summary}

The objective of the study was to determine the relationship between the variables academic selfconcept and academic motivation in young talented people from Puno. The methodology corresponds to the correlational type study, of non-experimental design and cross section; We worked with a population of 80 university beneficiaries of the scholarship program 18 from a private university in Puno, being $48.8 \%$ male and $51.3 \%$ female, to whom two documentary measurement instruments were applied: the self-concept sub-scale. AF5 academic and an academic motivation scale, both valid and reliable. The data were processed with the statistical package SPSS (Statistical Package for the Social Sciences) version 22.0 at a significance level of $5 \%$ and a confidence level of 95\%. The results according to Spearman's Rho correlation coefficient evidenced the existence of a moderate, direct and significant correlation between the research variables ( $\mathrm{Rho}=.497 ; \mathrm{p}=.000$ ) as well as in the intrinsic motivation dimensions (Rho $=.475, \mathrm{p}=.000)$ and extrinsic motivation $(\mathrm{Rho}=.485, \mathrm{p}=.000)$, whereas in Amotivación a low and negative but not significant correlation was found ( $\mathrm{Rho}=-.196, \mathrm{p}=.082$ ). It is concluded that the greater the academic self-concept, the greater the academic motivation.

Keywords: academic self-concept, academic motivation, young talent, national program Beca 18.

\section{Introducción}

Uno de los pilares para el desarrollo de una nación tiene que ver con la educación que reciben sus ciudadanos, que en la actualidad aun muestra desventajas de acceso para niños y adolescentes en el Perú (Instituto Nacional de Estadística e Informática [INEI], 2018). En tal sentido, la Organización de las Naciones Unidas (2015) presentó una agenda hasta el año 2030 en la cual resaltan 17 objetivos de desarrollo, precisamente el número cuatro implica reconocer que todo ciudadano debe tener acceso a posibilidades de aprendizaje permanente para adquirir los conocimientos y aptitudes necesarios para participar activamente en la sociedad, con esto 
claramente se busca erradicar la pobreza y garantizar una educación inclusiva y equitativa de calidad.

La realidad a nivel mundial, según estadística provistas por UNESCO (2017) aclara que el $28 \%$ de los niños no escolarizados, es decir 17 millones, no ha estudiado y probablemente nunca comenzará, además, el 38\% de los niños no escolarizados estudiaron alguna vez, pero desertaron del sistema educativo y el $34 \%$ tiene la probabilidad de estudiar, pero a mayor edad, por lo que hasta el año 2018 se consideraba que existían cerca de 264 millones de niños, adolescentes y jóvenes sin acceso a la educación. Por otra parte, en América Latina y el Caribe, haciendo énfasis en el contexto universitario, el Banco Mundial (2017) refiere que más de 20 millones de estudiantes universitarios de 10 mil instituciones provienen de hogares con menores recursos y no están preparados para enfrentar los desafíos de la vida universitaria. Aunado a esto, dicho informe revela que solo el $50 \%$ de estudiantes universitarios que inicia sus estudios se gradúa.

En el Perú, el Instituto Nacional de Estadística e Informática (INEI, 2019) informó resultados de la Encuesta Nacional de Hogares en el año 2018, donde los jóvenes de 15 a 29 años de edad superan los 8 millones de personas, de los cuales el 51.3\% corresponde a hombres y el $48.7 \%$ a mujeres; de estos, el $45.3 \%$ trabaja, el $11.3 \%$ trabajan y estudian, además, $26 \%$ solo estudian y $17.3 \%$ no trabaja ni estudia (NiNi). Así mismo, sobre el nivel educativo de la población de 15 a 29 años, un $7.5 \%$ tienen primaria o menor nivel, $54.7 \%$ educación secundaria, $14.9 \%$ logró estudiar superior no universitario, $22.5 \%$ logró estudiar superior universitaria y solo el $0.4 \%$ logró estudiar un posgrado.

Al presentar estos datos, no cabe duda de que el Perú al igual que otros países en América Latina y el mundo aun necesitan mejorar su sistema de educación y sobre todo el acceso de la misma a sus ciudadanos más vulnerables. Ante ello, hace algunos años el gobierno peruano instauró el Programa Nacional Beca 18 que es un ayuda integral dirigido a jóvenes de todo el país, especialmente aquellos que demuestra alto rendimiento académico, que están en condición de pobreza, pobreza extrema o vulnerabilidad social, provenientes de colegios públicos o privados. Estudios en dicha población revelan que esta oportunidad de subvención para nada es desaprovechada por los beneficiarios, que en su mayoría tienden a estar motivados hacia el estudio y sobre todo tener una definición positiva sobre su rendimiento académico (Carranza \& Apaza, 2015). Precisamente, las variables de la presente investigación tienen que ver con estos fenómenos, por un lado la motivación académica que según Herrera, Ramírez, Roa y Herrera (2004) es un proceso que activa, direcciona, y sobre todo persevera en una conducta para alcanzar un determinado objetivo, mientras que el auto concepto académico es la percepción que el individuo tiene de la calidad del desempeño de su rol como estudiante (Musitu \& García, 2014). Investigaciones con ambos constructos tanto a nivel internacional y nacional revelan la existencia de una dinamica de relaciòn entre ambas (Sosa, Sánchez \& Guerrero, 2016; Rey \& Barajas, 2014; Carranza \& Apaza, 2015; Ruiz, 2005).

Por todo lo mencionado, es que la presente investigación tiene el objetivo de determinar la relación entre motivación académica y autoconcepto académico en universitarios beneficiados por Beca 18 del departamento de Puno, que es una de las zonas con mayores de niveles de pobreza y grandes dificultades para el acceso a la educación en el Perú. 


\section{Método}

Esta investigación pertenece a un alcance correlacional, pues se tiene como propósito conocer la relación existente entre dos o más conceptos, categorías o variables en un contexto en particular. Así mismo, es de diseño no experimental y corte transversal ya que no existe manipulación activa de alguna variable y la medición en una muestra procede en un único momento en el tiempo (Hernandez, Fernandez, \& Baptista, 2014).

La población de estudio estuvo constituida por 80 estudiantes universitarios de ambos sexos beneficiarios del programa nacional Beca 18 matriculados en el VIII ciclo de escuelas profesionales como ingeniería ambiental, ingeniería de industrias alimentarias e ingeniería civil, provenientes de distintas provincias del departamento de Puno, matriculados en una universidad privada. En la presente investigación se prescindió de la muestra, según Supo (2014) se puede prescindir de una muestra cuando la población cumple con los tres criterios de exclusión como son el marco muestral, accesibilidad a los participantes y alcanzabilidad hacia los mismos, en este caso, se tiene identificados a cada uno de los encuestados, existen recursos para aplicar las encuestas en el $100 \%$ y es posible acceder a encuestar a todos los casos.

Para el recojo de la información se recurrió a instrumentos de medición documental como son la escala de motivación académica (EMA) (Núñez, Martín, Navarro, \& Grijalvo, 2006) conformada por 28 ítems y con forma de respuesta tipo Likert de siete puntos, desde $1=$ no se corresponde en absoluto, hasta $7=$ se corresponde totalmente. Por otra parte, la subescala de autoconcepto académico (AF5) de Musitu y García (2014) de 6 items con forma de respuesta también tipo Likert: nunca $(\mathrm{N})$, casi nunca $(\mathrm{CN})$, algunas veces (AV), casi siempre (CS), siempre (S). Ambos instrumentos válidos y confiables para los propósitos de la presente investigación.

El procedimiento para la ejecución de la investigación implicó en primera instancia solicitar la autorización del comité de ética de la institución superior, así también la coordinación para el día y la fecha del levantamiento de los datos. Durante la aplicación de las encuestas, antes de ser completadas, se solicitó el consentimiento informado firmado por los participantes, además se recalcó que la información brindada iba a tener un manejo estrictamente confidencial y solo para propósitos de la investigación. Después de la recolección, se procedió a calificar cada una de las encuestas, realizar control de calidad y tabular los datos en una matriz en Excel.

El análisis estadístico se realizó en el programa SPSS 25.0, obteniendo tablas de frecuencia y porcentaje y para la prueba de hipótesis se utilizó el coeficiente de correlación Rho de Spearman, adecuado para variables cualitativas. Se consideró un nivel de significancia del 5\% y nivel de confianza del 95\%. Finalmente, este estudio respeta la ética en la investigación, por ello se gestionó por los medios necesarios la autorización del comité de ética y el consentimiento informado por parte de los participantes.

\section{Resultados}

En cuanto a la validez y confiabilidad de los instrumentos, en ambos casos fueron sometidos a evaluación mediante juicio de 3 expertos cada uno, quienes luego de valorar la claridad de los ítems, su pertinencia, coherencia y congruencia, demostraron indicadores que según el coeficiente $\mathrm{V}$ de Aiken superan el corte .90, por lo tanto, se asume que en ambos casos se demuestra una alta validez de contenido. Por otro lado, en cuanto a la confiabilidad, se realizó una prueba piloto con 
30 estudiantes de VI semestre de otra universidad, de quienes surgieron indicadores según el coeficiente Alpha de Cronbach superan el corte .80, por lo que ambos demuestran una consistencia interna optima (Tabla 1).

Tabla 1

Validez de contenido y consistencia interna de los instrumentos de medición

\begin{tabular}{cccc}
\hline Escala & $\mathrm{N}^{\mathrm{o}}$ elementos & Alpha & V de Aiken \\
\hline Motivación académica & 28 & .962 & .985 \\
Autoconcepto académico & 6 & .922 & .978 \\
\hline
\end{tabular}

En cuanto a las características demográficas de la población, la tabla 2 da cuenta de que en mayor proporción existe universitarios de entre 20 y 21 años (72.5\%), ligeramente más mujeres (51.2\%) que varones $(48.8 \%)$, el $100 \%$ pertenecen al VIII ciclo académico, $43.8 \%$ estudia ingeniería civil, $45 \%$ ingeniería ambiental y $11.3 \%$ ingeniería de industrias alimentarias. En cuanto a religión, $52.5 \%$ son católicos, $40 \%$ adventistas, $3.8 \%$ evangélicos y $1.3 \%$ mormón; finalmente, el 5\% son de la costa, $83.8 \%$ de la sierra y $11.3 \%$ de la Selva.

En la misma tabla, el porcentaje más prevalente en cuanto a los niveles de motivación académica y las variables sociodemográficas se ubica en un nivel alto, como se percibe a continuación: entre 20 a 21 años de edad (41.3\%), género femenino (32.5\%), encuestados de ingeniería civil $(22.5 \%)$, en religión católicos $(28.7 \%)$, finalmente en relación a procedencia la Sierra $(45 \%)$.

Tabla 2

Niveles de motivación académica según variables sociodemográficas

\begin{tabular}{|c|c|c|c|c|c|c|c|c|c|}
\hline \multirow{3}{*}{ Variable } & \multirow{3}{*}{ Categoría } & \multicolumn{8}{|c|}{ Motivación académica } \\
\hline & & \multicolumn{2}{|l|}{ Bajo } & \multicolumn{2}{|c|}{ Moderada } & \multicolumn{2}{|c|}{ Alto } & \multicolumn{2}{|c|}{ Total } \\
\hline & & $\%$ & $\mathrm{f}$ & $\%$ & $\mathrm{f}$ & $\%$ & $\mathrm{f}$ & $\%$ & $\mathrm{f}$ \\
\hline \multirow[t]{3}{*}{ Edad } & 20 a 21 años & $7,5 \%$ & 6 & $23,8 \%$ & 19 & $41,3 \%$ & 33 & $72,5 \%$ & 58 \\
\hline & 22 a 23 años & $0,0 \%$ & 0 & $11,3 \%$ & 9 & $8,8 \%$ & 7 & $20,0 \%$ & 16 \\
\hline & 24 a 25 años & $0,0 \%$ & 0 & $5,0 \%$ & 4 & $2,5 \%$ & 2 & $7,5 \%$ & 6 \\
\hline \multirow[t]{2}{*}{ Sexo } & Masculino & $6,3 \%$ & 5 & $22,5 \%$ & 18 & $20,0 \%$ & 16 & $48,8 \%$ & 39 \\
\hline & Femenino & $1,3 \%$ & 1 & $17,5 \%$ & 14 & $32,5 \%$ & 26 & $51,2 \%$ & 41 \\
\hline Ciclo & Ciclo VIII & $7.5 \%$ & 6 & $40.0 \%$ & 32 & $52.5 \%$ & 42 & $100 \%$ & 80 \\
\hline \multirow[t]{3}{*}{ Carrera } & Civil & $5,0 \%$ & 4 & $16,3 \%$ & 13 & $22,5 \%$ & 18 & $43,8 \%$ & 35 \\
\hline & Ambiental & $2,5 \%$ & 2 & $22,5 \%$ & 18 & $20,0 \%$ & 16 & $45,0 \%$ & 36 \\
\hline & Industrias alimentarias & $0,0 \%$ & 0 & $1,3 \%$ & 1 & $10,0 \%$ & 8 & $11,3 \%$ & 9 \\
\hline \multirow[t]{5}{*}{ Religión } & Católico & $5,0 \%$ & 4 & $18,8 \%$ & 15 & $28,7 \%$ & 23 & $52,5 \%$ & 42 \\
\hline & Adventista & $2,5 \%$ & 2 & $18,8 \%$ & 15 & $18,8 \%$ & 15 & $40,0 \%$ & 32 \\
\hline & Evangélico & $0,0 \%$ & 0 & $1,3 \%$ & 1 & $2,5 \%$ & 2 & $3,8 \%$ & 3 \\
\hline & Mormón & $0,0 \%$ & 0 & $0,0 \%$ & 0 & $1,3 \%$ & 1 & $1,3 \%$ & 1 \\
\hline & Otros & $0,0 \%$ & 0 & $1,3 \%$ & 1 & $1,3 \%$ & 1 & $2,5 \%$ & 2 \\
\hline \multirow[t]{3}{*}{ Procedencia } & Costa & $1,3 \%$ & 1 & $1,3 \%$ & 1 & $2,5 \%$ & 2 & $5,0 \%$ & 4 \\
\hline & Sierra & $6,3 \%$ & 5 & $32,5 \%$ & 26 & $45,0 \%$ & 36 & $83,8 \%$ & 67 \\
\hline & Selva & $0,0 \%$ & 0 & $6,3 \%$ & 5 & $5,0 \%$ & 4 & $11,3 \%$ & 9 \\
\hline
\end{tabular}


Continuando, según la tabla 3 el porcentaje más prevalente entre los niveles de autoconcepto académico y las variables sociodemográficas se ubica también en el nivel alto, como se percibe a continuación: entre 20 a 21 años de edad (42.5\%), género femenino (28.7\%), encuestados de ingeniería ambiental (25.0\%), religión, otra vez católicos (32.5\%), finalmente en relación a procedencia la sierra $(45 \%)$.

Tabla 3

Niveles de autoconcepto académico según variables sociodemográficas

\begin{tabular}{|c|c|c|c|c|c|c|c|c|c|}
\hline \multirow{3}{*}{ Variable } & \multirow{3}{*}{ Categorías } & \multicolumn{8}{|c|}{ Autoconcepto académico } \\
\hline & & \multicolumn{2}{|c|}{ Bajo } & \multicolumn{2}{|c|}{ Moderada } & \multicolumn{2}{|c|}{ Alto } & \multicolumn{2}{|c|}{ Total } \\
\hline & & $\%$ & $\mathrm{f}$ & $\%$ & $\mathrm{f}$ & $\%$ & $\mathrm{f}$ & $\%$ & $\bar{f}$ \\
\hline \multirow[t]{3}{*}{ Edad } & 20 a 21 años & $16,3 \%$ & 13 & $13,8 \%$ & 11 & $42,5 \%$ & 34 & $72,5 \%$ & 58 \\
\hline & 22 a 23 años & $7,5 \%$ & 6 & $3,8 \%$ & 3 & $8,8 \%$ & 7 & $20,0 \%$ & 16 \\
\hline & 24 a 25 años & $3,8 \%$ & 3 & $0,0 \%$ & 0 & $3,8 \%$ & 3 & $7,5 \%$ & 6 \\
\hline \multirow[t]{2}{*}{ Sexo } & Masculino & $12,5 \%$ & 10 & $10,0 \%$ & 8 & $26,3 \%$ & 21 & $48,8 \%$ & 39 \\
\hline & Femenino & $15,0 \%$ & 12 & $7,5 \%$ & 6 & $28,7 \%$ & 23 & $51,2 \%$ & 41 \\
\hline Ciclo & Ciclo VIII & $27,5 \%$ & 22 & $17,5 \%$ & 14 & $55,0 \%$ & 44 & $100 \%$ & 80 \\
\hline \multirow[t]{3}{*}{ Carrera } & Civil & $12,5 \%$ & 10 & $10,0 \%$ & 8 & $21,3 \%$ & 17 & $43,8 \%$ & 35 \\
\hline & Ambiental & $13,8 \%$ & 11 & $6,3 \%$ & 5 & $25,0 \%$ & 20 & $45,0 \%$ & 36 \\
\hline & Industrias alimentarias & $1,3 \%$ & 1 & $1,3 \%$ & 1 & $8,8 \%$ & 7 & $11,3 \%$ & 9 \\
\hline \multirow[t]{5}{*}{ Religión } & Católico & $12,5 \%$ & 10 & $7,5 \%$ & 6 & $32,5 \%$ & 26 & $52,5 \%$ & 42 \\
\hline & Adventista & $12,5 \%$ & 10 & $7,5 \%$ & 6 & $20,0 \%$ & 16 & $40,0 \%$ & 32 \\
\hline & Evangélico & $0,0 \%$ & 0 & $1,3 \%$ & 1 & $2,5 \%$ & 2 & $3,8 \%$ & 3 \\
\hline & Mormón & $0,0 \%$ & 0 & $1,3 \%$ & 1 & $0,0 \%$ & 0 & $1,3 \%$ & 1 \\
\hline & Otros & $2,5 \%$ & 2 & $0,0 \%$ & 0 & $0,0 \%$ & 0 & $2,5 \%$ & 2 \\
\hline \multirow[t]{3}{*}{ Procedencia } & Costa & $1,3 \%$ & 1 & $0,0 \%$ & 0 & $3,8 \%$ & 3 & $5,0 \%$ & 4 \\
\hline & Sierra & $22,5 \%$ & 18 & $16,3 \%$ & 13 & $45,0 \%$ & 36 & $83,8 \%$ & 67 \\
\hline & Selva & $3,8 \%$ & 3 & $1,3 \%$ & 1 & $6,3 \%$ & 5 & $11,3 \%$ & 9 \\
\hline
\end{tabular}

En cuestión de las frecuencias y porcentajes para las variables de estudio, la tabla 4 revela que el $55.0 \%$ de los estudiantes presenta un nivel alto de autoconcepto académico, un $27.5 \%$ un nivel bajo y solo $17.5 \%$ presenta un nivel moderado. Por otra parte, respecto a la variable motivación académica, el $52.5 \%$ de los estudiantes presenta un nivel alto y un $40.0 \%$ un nivel moderado y un $7.5 \%$ un nivel bajo. 
Tabla 4

Niveles de autoconcepto académico y motivación académica

\begin{tabular}{llcc}
\hline Variable & Categoría & $\mathrm{f}$ & $\%$ \\
\hline \multirow{2}{*}{ Autoconcepto académico } & Bajo & 22 & 27.5 \\
& Moderado & 14 & 17.5 \\
& Alto & 44 & 55.0 \\
& Total & 80 & 100.0 \\
Motivación académica & Bajo & 6 & 7.5 \\
& Moderado & 32 & 40.0 \\
& Alto & 42 & 52.5 \\
& Total & 80 & 100.0 \\
\hline
\end{tabular}

En cuanto a la prueba de hipótesis, según la tabla 5 existen correlaciones directas y significativas entre autoconcepto académico con motivación académica (Rho $=.497, \mathrm{p}=.000$ ), con la dimensión motivación intrínseca $(\mathrm{Rho}=.475, \mathrm{p}=.000)$, con la dimensión motivación extrinseca $($ Rho $=.485, \mathrm{p}=.000)$; en cambio, una correlación baja mas no significativa con la dimensión amotivación (Rho $=-.196, \mathrm{p}=.082)$. Esto quiere decir que tanto en la hipótesis general como las primeras dos especificas se acepta la Ha, mientras que en la tercera especifica se acepta la Ho.

\section{Tabla 5}

Coeficientes de correlación entre autoconcepto académico y las dimensiones de motivación académica

\begin{tabular}{lcc}
\hline Adicción a redes sociales y sus dimensiones & \multicolumn{2}{c}{ Autoceoncepto académico } \\
\hline & rho & $\mathrm{p}$ \\
\cline { 2 - 3 } Motivación académica & $.497^{* *}$ & 0.000 \\
Motivación extrínseca & $.485^{* *}$ & 0.000 \\
Motivación intrínseca & $.475^{* *}$ & 0.000 \\
Amotivación & $-.196^{\mathrm{ns}}$ & 0.082
\end{tabular}

** La correlación es significante a nivel 0.01

* La correlación es significante a nivel 0.05

\section{Discusión}

Los hallazgos permiten confirmar la hipótesis de esta investigación, es decir que los niveles de autoconcepto académico (AA) se asocian con los niveles de motivación académica (MA) en universitarios de Beca 18. El análisis estadístico permitió establecer la existencia de correlaciones positivas entre la variable AA con la dimensión motivación intrínseca, dimensión motivación extrínseca y la variable MA; en cambio, la correlación no es significativa con la dimensión amotivación. En base a esto, se puede asumir que en la medida que los estudiantes tengan una reflexión positiva de sí mismo en cuanto a su rendimiento académico (Montoya, Pinilla \& Dussan, 2018), entonces mayor será la motivación con la que enfrenten tareas y retos en la universidad. Sin embargo, también es necesario reconocer que los motivos de un estudiante para destacar en 
el contexto universitario implica otras variables, no obstante, los resultados encontrados permiten asumir que el autoconcepto académico incide sobre el (Urquijo, 2002).

Estos resultados coinciden con lo reportado en la literatura científica; por ejemplo, con el trabajo de Carranza y Apaza (2015) quienes también abordaron el AA y MA en un grupo de universitarios de Beca 18 pero de la Región San Martín. Sus resultados evidenciaron asociaciones significativas entre ambas variables, un hecho similar al presente trabajo, no obstante, el estudio mencionado revela que amotivación también evidencia asociación con AA, un resultado diferente a la presente. Por otro lado Guay, Ratelle, Roy y Litalien (2010) en estudio realizado con adolescentes de nivel secundario halló bajo un modelo de ecuaciones estructurales que la MA autonoma media la relación AA - rendimiento academico, un planteamiento distinto a la presente, donde se propone que es el AA el que determinar niveles de MA. Finalmente, aparece el estudio de Suarez-Alvarez, Fernández-Alonso y Muñiz (2014) quienes estudiaron también a adolescentes, en este caso de segundo grado de educación secundaria, y hallaron que aunque AA no es un predictor de la MA, si demuestra ser una variable con mayor poder predictivo del desempeño academico, un resultado contrario al nuestro.

Otro aspecto relevante dentro de los resultados tiene que ver con el hecho de que los universitarios de Beca 18 de Puno, demuestran en su mayoría (más del 50\%) tener un nivel de AA alto, de igual forma en MA (más del 50\% con nivel alto). Tomando en cuenta los antecedentes estos datos parecen ser normales en este tipo de estudiantes, más aún cuando analizamos la situación de cada becario, pues cerca de las $3 / 4$ partes provienen de las provincias del departamento de Puno, y muchos de ellos son de familias de nivel socioeconómico muy bajo y con grandes limitaciones de acceso a la educación; lo cual, explica muy bien su esfuerzo y dedicación (Salinas, Hernández \& Barboza-Palomino, 2017).

Aunado a esto, la dinámica que surge al reconocerse a si mismo como un buen estudiante y a consecuencia de ello, enfrentar, desafiar e impulsarse a generar logros académicos, es lo que Gonzales, Leal, Segovia \& Arancibia (2012) refieren como autoconcepto y talento: una relación que favorece el logro académico.

En suma, la literatura científica da cuenta de la importancia de la motivación para alcanzar el logro académico en el contexto universitario (Aldanas, Rivero \& Garnache, 2016), de donde surgen los futuros profesionales para un país, no obstante, esta relación según los estudios encontrados no es lineal, por lo que existen otros factores implicados en el buen rendimiento, sin embargo, en base a resultados como los de la presente investigación es que existe una fuerte corriente en la comunidad científica para asumir que el AA es un factor determinante al momento de que un estudiante busque un óptimo rendimiento académico en la universidad (Han, 2019; Jansen, Schroeders, Lüdtke, 2014).

El estudio tuvo limitaciones ya que consideró a jóvenes talento de una sola universidad de la ciudad de Juliaca, asimismo, por su naturaleza (diseño transversal y alcance correlacional), no fue posible analizar relaciones causales. En este sentido, se recomienda que los próximos estudios sean de carácter experimental o longitudinal; además, sería pertinente realizar investigaciones que analicen la MA con otras variables como la autoeficacia académica, autorregulación, satisfacción con vida estudiantil. 
Se concluye que en la medida que un estudiante universitario tenga una reflexión y conciencia positiva acerca de sus capacidades involucradas en el rendimiento académico, entonces mayor es la capacidad para impulsarse hacia el logro académico en la universidad, una situación evidente en estudiantes becarios.

\section{Referencias}

Aldanás, M., Rivero, M., \& Garnache, A. (2016). La motivación en el rendimiento académico de los estudiantes de medicina. Médica Electrónica, 38(6), 910-915. Recuperado de http://scielo.sld.cu/scielo.php?script=sci_arttext\&pid=S1684-18242016000600013

Banco Mundial. (2017). Graduarse: solo la mitad lo logra en América Latina.

Carranza, R. F., \& Apaza, E. E. (2015). Autoconcepto académico y motivación académica en jóvenes talento de una universidad privada de Tarapoto. Propósitos y Representaciones, 3(1), 249-263.

Gonzáles, M., Leal, D., Segovia, C., \& Arancibia, V. (2012). Autoconcepto y Talento: Una relación que favorece el logro académico. PSYKHE, 21(1), 37-53. Recuperado de http://dx.doi.org/10.4067/S0718-22282012000100003

Guay, F., Ratelle, C. F., Roy, A., \& Litalien, D. (2010). Academic self-concept, autonomous academic motivation, and academic achievement: Mediating and additive effects. Learning and Individual Differences, 20(6), 644-653. https://doi.org/10.1016/j.lindif.2010.08.001

Han, F. (2019). Relaciones longitudinales entre el autoconcepto académico y el rendimiento académico. Psicodidáctica, 24(2), 95 - 102. Recuperado de https://www.sciencedirect.com/science/article/abs/pii/S1136103418302041

Hernandez, R., Fernandez, C., \& Baptista, P. (2014). Metodologia de la investigación. Mexico: Mc Graw Hill.

Herrera, F., Ramírez, I., Roa, J., \& Herrera, I. (2004). Tratamiento de las creencias motivacionales en contextos educativos pluriculturales. Revista Iberoamericana de Educación, 34(1), 1-21. https://doi.org/10.35362/rie3412885

INEI. (2018). Perú Resultados definitivos. In INEI. Retrieved from https:// www.inei.gob.pe/media/MenuRecursivo/publicaciones_digitales/Est/L ib1544/

INEI. (2019). Indicadores de Educación por Departamento 2008 - 2018. In INEI. Retrieved from https://www.inei.gob.pe/media/MenuRecursivo/publicaciones_digitales/Est/Lib1680/libro. pdf

Jansen, M., Schroeders, U., \& Ludtke, O. (2014). Academic self-concept in science: Multidimensionality, relations to achievement measures, and gender differences. ELSEVIER, 30, 11-21. Obtenido de https://doi.org/10.1016/j.lindif.2013.12.003

Montoya, D., Pinilla, V., \& Dussán, C. (2018). Caracterización del autoconcepto en una muestra de estudiantes universitarios de algunos programas de pregrado de la ciudad de Manizales. Psicogente, 21(29), 162 - 182. Obtenido de http://doi.org/10.17081/psico.21.39.2829

Musitu, G., \& García, F. (2014). Manual AF-5 Autoconcepto forma 5. En TEA.

Núñez, J., Martín, J., Navarro, J., \& Grijalvo, F. (2006). Validación de la escala de motivación educativa (EME) en paraguay. Interamerican Journal of Psychology, 40(3), 391-398.

Organización de las Naciones Unidas. (2015). Transformar nuestro mundo: la Agenda 2030 para el Desarrollo Sostenible. En Organización de las Naciones Unidas.

Rey, H., \& Barajas, A. (2014). La influencia del autoconcepto académico en el rendimiento escolar de las asignaturas de lengua castellana y matemáticas. caso de los estudiantes del grado ocho tres de la Institución Educativa Técnico Rafael García -Herreros del Municipio 
de Bucaramanga. Universidad del Tolima.

Ruiz, F. (2005). Relación entre la motivación de logro académico , la autoeficacia y la disposición para la realización de una tesis. Persona, (8), 145-170.

Salinas, D., Hérnandez, A., \& Barboza - Palomino, M. (2017). Condición de becario y rendimiento académico en estudiantes de una universidad peruana. Electrónica de Investigación Educativa, 19(4), 124-133. Recuperado de https://doi.org/10.24320/redie.2017.19.4.1348

Sosa, D., Sánchez, S., \& Guerrero, E. (2016). Autoconcepto académico: modalidades de escolarización, repeticiones de curso y sexo: Campo Abierto, 35(2), 69-82. Obtenido de https://relatec.unex.es/revistas/index.php/campoabierto/article/view/2214/pd f_28

Suarez-Alvarez, J., Fernández-Alonso, R., \& Muñiz, J. (2014). Autoconcepto, motivación, expectativas y nivel socioeconómico como predictores del desempeño académico en matemáticas. Learning and Individual Differences, 30(2), 118-123.

Supo, J. (2014). Cómo elegir una muestra. Arequipa: Bioestadistico EIRL.

UNESCO. (2017). Reducir la pobreza en el mundo gracias a la enseñanza primaria y secundaria universal.

Urquijo, S. (2002). Auto-concepto y desempeño académico en adolescentes. Relaciones con sexo, edad e institución. Psico - USF, 7(2), 211-218. Recuperado de http://pepsic.bvsalud.org/pdf/psicousf/v7n2/v7n2a10.pdf 


\section{ANEXOS}

Tabla 1

Validez de contenido y consistencia interna de los instrumentos de medición

\begin{tabular}{cccc}
\hline Escala & $\mathrm{N}^{\text {o }}$ elementos & Alpha & V de Aiken \\
\hline Motivación académica & 28 & .962 & .985 \\
Autoconcepto académico & 6 & .922 & .978 \\
\hline
\end{tabular}

Tabla 2

Niveles de motivación académica según variables sociodemográficas

\begin{tabular}{|c|c|c|c|c|c|c|c|c|c|}
\hline \multirow{3}{*}{ Variable } & \multirow{3}{*}{ Categoría } & \multicolumn{8}{|c|}{ Motivación académica } \\
\hline & & \multicolumn{2}{|c|}{ Bajo } & \multicolumn{2}{|c|}{ Moderada } & \multicolumn{2}{|c|}{ Alto } & \multicolumn{2}{|c|}{ Total } \\
\hline & & $\%$ & $f$ & $\%$ & $\mathrm{f}$ & $\%$ & $\mathrm{f}$ & $\%$ & $f$ \\
\hline \multirow[t]{3}{*}{ Edad } & 20 a 21 años & $7,5 \%$ & 6 & $23,8 \%$ & 19 & $41,3 \%$ & 33 & $72,5 \%$ & 58 \\
\hline & 22 a 23 años & $0,0 \%$ & 0 & $11,3 \%$ & 9 & $8,8 \%$ & 7 & $20,0 \%$ & 16 \\
\hline & 24 a 25 años & $0,0 \%$ & 0 & $5,0 \%$ & 4 & $2,5 \%$ & 2 & $7,5 \%$ & 6 \\
\hline \multirow[t]{2}{*}{ Sexo } & Masculino & $6,3 \%$ & 5 & $22,5 \%$ & 18 & $20,0 \%$ & 16 & $48,8 \%$ & 39 \\
\hline & Femenino & $1,3 \%$ & 1 & $17,5 \%$ & 14 & $32,5 \%$ & 26 & $51,2 \%$ & 41 \\
\hline Ciclo & Ciclo VIII & $7.5 \%$ & 6 & $40.0 \%$ & 32 & $52.5 \%$ & 42 & $100 \%$ & 80 \\
\hline \multirow[t]{3}{*}{ Carrera } & Civil & $5,0 \%$ & 4 & $16,3 \%$ & 13 & $22,5 \%$ & 18 & $43,8 \%$ & 35 \\
\hline & Ambiental & $2,5 \%$ & 2 & $22,5 \%$ & 18 & $20,0 \%$ & 16 & $45,0 \%$ & 36 \\
\hline & Industrias alimentarias & $0,0 \%$ & 0 & $1,3 \%$ & 1 & $10,0 \%$ & 8 & $11,3 \%$ & 9 \\
\hline \multirow[t]{5}{*}{ Religión } & Católico & $5,0 \%$ & 4 & $18,8 \%$ & 15 & $28,7 \%$ & 23 & $52,5 \%$ & 42 \\
\hline & Adventista & $2,5 \%$ & 2 & $18,8 \%$ & 15 & $18,8 \%$ & 15 & $40,0 \%$ & 32 \\
\hline & Evangélico & $0,0 \%$ & 0 & $1,3 \%$ & 1 & $2,5 \%$ & 2 & $3,8 \%$ & 3 \\
\hline & Mormón & $0,0 \%$ & 0 & $0,0 \%$ & 0 & $1,3 \%$ & 1 & $1,3 \%$ & 1 \\
\hline & Otros & $0,0 \%$ & 0 & $1,3 \%$ & 1 & $1,3 \%$ & 1 & $2,5 \%$ & 2 \\
\hline \multirow[t]{3}{*}{ Procedencia } & Costa & $1,3 \%$ & 1 & $1,3 \%$ & 1 & $2,5 \%$ & 2 & $5,0 \%$ & 4 \\
\hline & Sierra & $6,3 \%$ & 5 & $32,5 \%$ & 26 & $45,0 \%$ & 36 & $83,8 \%$ & 67 \\
\hline & Selva & $0,0 \%$ & 0 & $6,3 \%$ & 5 & $5,0 \%$ & 4 & $11,3 \%$ & 9 \\
\hline
\end{tabular}


Tabla 3

Niveles de autoconcepto académico según variables sociodemográficas

\begin{tabular}{|c|c|c|c|c|c|c|c|c|c|}
\hline \multirow{3}{*}{ Variable } & \multirow{3}{*}{ Categorías } & \multicolumn{8}{|c|}{ Autoconcepto académico } \\
\hline & & \multicolumn{2}{|c|}{ Bajo } & \multicolumn{2}{|c|}{ Moderada } & \multicolumn{2}{|c|}{ Alto } & \multicolumn{2}{|c|}{ Total } \\
\hline & & $\%$ & $\mathrm{f}$ & $\%$ & $f$ & $\%$ & $\mathrm{f}$ & $\%$ & $\mathrm{f}$ \\
\hline \multirow[t]{3}{*}{ Edad } & 20 a 21 años & $16,3 \%$ & 13 & $13,8 \%$ & 11 & $42,5 \%$ & 34 & $72,5 \%$ & 58 \\
\hline & 22 a 23 años & $7,5 \%$ & 6 & $3,8 \%$ & 3 & $8,8 \%$ & 7 & $20,0 \%$ & 16 \\
\hline & 24 a 25 años & $3,8 \%$ & 3 & $0,0 \%$ & 0 & $3,8 \%$ & 3 & $7,5 \%$ & 6 \\
\hline \multirow[t]{2}{*}{ Sexo } & Masculino & $12,5 \%$ & 10 & $10,0 \%$ & 8 & $26,3 \%$ & 21 & $48,8 \%$ & 39 \\
\hline & Femenino & $15,0 \%$ & 12 & $7,5 \%$ & 6 & $28,7 \%$ & 23 & $51,2 \%$ & 41 \\
\hline Ciclo & Ciclo VIII & $27,5 \%$ & 22 & $17,5 \%$ & 14 & $55,0 \%$ & 44 & $100 \%$ & 80 \\
\hline \multirow[t]{3}{*}{ Carrera } & Civil & $12,5 \%$ & 10 & $10,0 \%$ & 8 & $21,3 \%$ & 17 & $43,8 \%$ & 35 \\
\hline & Ambiental & $13,8 \%$ & 11 & $6,3 \%$ & 5 & $25,0 \%$ & 20 & $45,0 \%$ & 36 \\
\hline & Industrias alimentarias & $1,3 \%$ & 1 & $1,3 \%$ & 1 & $8,8 \%$ & 7 & $11,3 \%$ & 9 \\
\hline \multirow[t]{5}{*}{ Religión } & Católico & $12,5 \%$ & 10 & $7,5 \%$ & 6 & $32,5 \%$ & 26 & $52,5 \%$ & 42 \\
\hline & Adventista & $12,5 \%$ & 10 & $7,5 \%$ & 6 & $20,0 \%$ & 16 & $40,0 \%$ & 32 \\
\hline & Evangélico & $0,0 \%$ & 0 & $1,3 \%$ & 1 & $2,5 \%$ & 2 & $3,8 \%$ & 3 \\
\hline & Mormón & $0,0 \%$ & 0 & $1,3 \%$ & 1 & $0,0 \%$ & 0 & $1,3 \%$ & 1 \\
\hline & Otros & $2,5 \%$ & 2 & $0,0 \%$ & 0 & $0,0 \%$ & 0 & $2,5 \%$ & 2 \\
\hline \multirow[t]{3}{*}{ Procedencia } & Costa & $1,3 \%$ & 1 & $0,0 \%$ & 0 & $3,8 \%$ & 3 & $5,0 \%$ & 4 \\
\hline & Sierra & $22,5 \%$ & 18 & $16,3 \%$ & 13 & $45,0 \%$ & 36 & $83,8 \%$ & 67 \\
\hline & Selva & $3,8 \%$ & 3 & $1,3 \%$ & 1 & $6,3 \%$ & 5 & $11,3 \%$ & 9 \\
\hline
\end{tabular}

Tabla 4

Niveles de autoconcepto académico y motivación académica

\begin{tabular}{llcc}
\hline Variable & Categoría & $\mathrm{f}$ & $\%$ \\
\hline Autoconcepto académico & Bajo & 22 & 27.5 \\
& Moderado & 14 & 17.5 \\
& Alto & 44 & 55.0 \\
\multirow{3}{*}{ Motivación académica } & Total & 80 & 100.0 \\
& Bajo & 6 & 7.5 \\
& Moderado & 32 & 40.0 \\
& Alto & 42 & 52.5 \\
& Total & 80 & 100.0 \\
\hline
\end{tabular}

Tabla 5

Coeficientes de correlación entre autoconcepto académico y las dimensiones de motivación académica

Adicción a redes sociales y sus dimensiones $\quad$ Autoceoncepto académico 
Motivación académica

Motivación extrínseca

Motivación intrínseca

Amotivación
$.497 * *$

$.485^{* *}$

$475^{* *}$

$-.196^{\mathrm{ns}}$
0.000

0.000

** La correlación es significante a nivel 0.01

* La correlación es significante a nivel 0.05 